\title{
Aprendizaje inverso en formación profesional: opiniones de los estudiantes
}

\section{Flipped Classroom in Professional Training: Students' opinions}

\author{
David Fernández-Gámez \\ Escuela de Emergencias SAMU (davidfdezgamez@gmail.com) \\ María Dolores Guerra-Martín \\ Departamento de Enfermería. Universidad de Sevilla, España (guema@us.es)
}

Recibido el 19 de noviembre de 2015; revisado el 13 de enero de 2016; aceptado el 2 de marzo de 2016 ; publicado el 1 de junio de 2016

\section{RESUMEN:}

El aprendizaje inverso o flipped classroom es una metodología de aprendizaje que fomenta el trabajo autónomo del alumnado, transfiriendo fuera del aula el trabajo de determinados procesos de aprendizaje. Requiere de una tecnología y diseño pedagógico centrado en promover potencialidades de aprendizaje en los estudiantes. Nos propusimos como objetivos poner en marcha el aprendizaje inverso en el aula durante el curso 2014/2015 y conocer las opiniones del proceso de aprendizaje de los alumnos de la asignatura Dotación Sanitaria dentro del Ciclo Formativo de Grado Medio de Técnico en Emergencias Sanitarias en Sevilla (España). Tras la finalización del curso se realizó un estudio descriptivo y transversal, con una muestra de trece alumnos matriculados $(100 \%)$. Se realizó un cuestionario de elaboración propia conformado por doce preguntas, con seis opciones de respuesta tipo Likert. La recogida de datos se realizó en el aula, de manera autoadministrada. En los resultados obtenidos, el $100 \%$ de los alumnos estuvo de acuerdo y totalmente de acuerdo con que el aprendizaje inverso les permite tener más tiempo para resolver las dudas en clase. Además, ningún estudiante ha valorado negativamente ningún ítem, ni ha dejado ninguno sin contestar. Como conclusión, decir que el alumnado reconoce que el aprendizaje inverso es más motivador que el tradicional, ya que promueve la investigación y reflexión, y mejora el rendimiento formativo. Para llevar a cabo este aprendizaje inverso, se necesitan una tecnología apropiada y un diseño pedagógico centrado en promover el trabajo autónomo del alumnado.

PALABRAS CLAVE: APRENDIZAJE INVERSO, EDUCACIÓN PROFESIONAL, APRENDIZAJE SEMIPRESENCIAL.

\section{ABSTRACT:}

Flipped classroom is a learning methodology that encourages the independent work of students, transferring the work of certain learning processes outside the classroom. It requires a technology and pedagogical design focused on promoting potentials of learning in students. Our aims were starting flipped classroom during the academic year 2014 / 2015 and hear the views of the learning process of the students of the subject Dotación sanitaria within the training in health emergencies in Seville (Spain). Once the course finished, it was made a descriptive and transversal study, with a sample of thirteen students enrolled $(100 \%)$. It was made a homemade questionnaire consisting of twelve questions, with six options of likert-type response. Data collection was carried out in the classroom, self-administered way. In the results, $100 \%$ of the students was agreement and agree with that flipped classroom allows them to have more time to resolve questions in class. Furthermore, any item was negatively appreciated by students and all questions were answered by them. As conclusion, students recognize that reverse learning is more motivating 
than traditional, one since it promotes research and reflection, and improves the training performance. In order to perform this inverse learning, an appropriate technology and pedagogical design focused on promoting independent work of students are required.

\section{KEYWORDS:}

\section{FLIPPED CLASSROOM, EDUCATION, PROFESSIONAL, BLENDED LEARNING.}

\section{INTRODUCCION}

\subsection{Planteamiento del problema}

El déficit de atención lleva al aburrimiento en clase de los alumnos, disminuyendo progresivamente el interés y la concentración. Sin motivación, el alumnado no presenta un comportamiento adaptativo ante nuevos aprendizajes, habiendo un déficit conductual en la consecución de metas (Lozano, 2000). En el aprendizaje, el alumnado debe ser capaz de reflexionar y asimilar los conocimientos y habilidades propuestos por el docente, cuyo rol será el de facilitador y orientador (Tourón y Santiago, 2015). Por su parte, el alumno pasará por fases intermedias en la reorganización del conocimiento, necesitando una actitud favorable, expectativas y motivación para aprender. La motivación es un elemento esencial para la marcha del aprendizaje y es inherente a la posibilidad de otorgar sentido y significado al conocimiento. Sin motivación, el alumnado no realizará un trabajo adecuado, no solo el de aprender un concepto, sino el de poner en marcha estrategias que le permitan resolver problemas similares a lo aprendido (Núñez, 2009).

Una estrategia democrática y colaborativa que aumenta y mantiene la motivación es el uso pedagógico de las Tecnologías de la Información y Comunicación (TIC) (Guerra-Martín, Lima-Serrano, Zambrano-Domínguez y Fernández-Rodríguez, 2013; Guerra-Martín, Lima-Serrano, ZambranoDomínguez y Lima-Rodríguez, 2014). La implementación de este uso en los centros educativos promueve una enseñanza personalizada y constructivista, que otorga al alumnado competencias tecnológicas que la actual Sociedad del Conocimiento está demandando (Gómez y Vidangos, 2010). Teniendo en cuenta esta demanda social y el aumento de la motivación con el uso de las TIC, se utiliza la metodología de aprendizaje inverso o flipped classroom como apoyo tecnológico innovador en el desarrollo del proceso de enseñanza - aprendizaje. Con el aprendizaje inverso se pretende mejorar la comprensión conceptual y capacitar al alumnado en habilidades tecnológicas, integrándole en la Sociedad del Conocimiento y motivándole para que sea capaz de resolver problemas desde una perspectiva constructivista (Tourón y Santiago, 2013). El uso de las TIC por parte del profesorado debe ser pedagógico, por lo que no es suficiente tener el mero conocimiento técnico de estas tecnologías, sino que el profesorado debe ser capaz de manejarlo adecuadamente y de adaptarlo al estilo de aprendizaje de los estudiantes (Guerra-Martín et al., 2013; Guerra-Martín et al., 2014).

\subsection{Antecedentes y Fundamentación teórica}

La motivación sirve para alcanzar objetivos de aprendizaje y satisfacer necesidades de los alumnos (Núñez, 2009). Existe una variabilidad de definiciones de motivación. Una de ellas, define a la motivación como la fuerza que impulsa a la persona a adoptar una conducta determinada (De la Torre, 2000). La Real Academia Española (2011), la define como un ensayo mental preparatorio de una acción para animar o animarse a ejecutarla con interés y diligencia. La motivación estimula al individuo a la búsqueda continua de mejores situaciones a fin de realizarse personalmente e integrarse en la sociedad (García y Doménech, 2002).

Hoy en día aprender no significa únicamente memorizar la información, sino que es necesario también comprender la nueva información, analizarla, considerar relaciones con situaciones conocidas, sintetizar los nuevos conocimientos e integrarlos con los saberes previos para lograr su aplicación en diferentes contextos (Julián y Narváez, 2010; Guerra-Martín et al., 2014). Gracias a la motivación, la predisposición del alumnado a aprender aumenta, y se empiezan a observar otras formas de aprendizaje aparte del formal (Marcelo, 2011). Aprender, por tanto, supone un proceso complejo que va más allá de la simple acumulación de información. Se aprende relacionando el conocimiento nuevo con el que ya se posee. No hay una única ruta de aprendizaje, sino que las posibilidades pueden ser múltiples, dependiendo del contexto, del tiempo, de los objetivos y contenidos de aprendizaje y, sobre todo, de las características, intereses y necesidades de las personas implicadas en la formación (Amador, Monreal y Marco, 2001; (Guerra-Martín et al., 2014).

Aunque tengamos alumnos con unas necesidades formativas similares, el estilo de aprendizaje puede ser muy diferente. Este estilo es analizado por autores que han desarrollado sus respectivas teorías de aprendizaje. Entre las teorías de aprendizaje que integran las TIC en el proceso de aprendizaje 
destaca el conectivismo. Esta teoría, desarrollada en la era digital tras el análisis de las limitaciones del conductismo, el cognitivismo y el constructivismo, explica el efecto que la tecnología tiene sobre la manera en que actualmente vivimos, nos comunicamos y aprendemos (Siemens, 2004). Si aplicamos las TIC al proceso de enseñanzaaprendizaje, podremos observar que el alumnado dispondrá de diferentes fuentes de información, y aprenderá de una manera informal de las opiniones de sus compañeros, profesorado y personas que publican en sitios web (Marcelo, 2011). Esto es un aprendizaje autónomo y riguroso, llamado aprendizaje autodirigido o self-directed learning (Marcelo, 2010). El aprendizaje autónomo se adapta al estilo de aprendizaje, en contra de la enseñanza expositiva que no fomenta el aprendizaje reflexivo ni por descubrimiento (Gureckis y Markant, 2012).

Cada persona utiliza diferentes estrategias para recopilar, interpretar, organizar y pensar sobre la nueva información, o lo que es lo mismo, presenta diferentes estilos de aprendizaje (Nicasio, 2001). En este sentido, es importante resaltar que en la profesionalización e inserción profesional de los estudiantes, se ha de fomentar el aprendizaje integrativo de las competencias tanto académicas como profesionales, y se debe fomentar el aprendizaje integrativo de ambas competencias (Isus y Roure, 2012; Guerra-Martín, 2014).

Aprender significa modificar los propios esquemas cognitivos, es decir, el aprendizaje debe ser significativo (Moreira, 2012). Todo aprendizaje parte de unos conocimientos previos. Para que el aprendizaje sea significativo, es preciso que: 1. El contenido propuesto como objeto de aprendizaje esté bien organizado, para que facilite al alumno su asimilación, mediante el establecimiento de relaciones entre aquél y los conocimientos que ya posee. 2. Los conceptos sigan una secuencia lógica y ordenada. 3. El alumno manifieste predisposición ante el aprendizaje propuesto. Por tanto, debe estar motivado para ello, tener interés y creer que puede hacerlo. 4. El alumno cuente con los conocimientos previos necesarios para enlazar los nuevos aprendizajes propuestos. Esto quiere decir que se necesita una base previa suficiente que establezca las relaciones necesarias para aprender (Ausubel, 1986).

Desde la perspectiva pedagógica de Ausubel (1986), podemos observar que las condiciones que requieren el aprendizaje significativo, pueden desarrollarse en entornos virtuales de aprendizaje. A través de las TIC, el alumnado es capaz de identificar su estilo de aprendizaje y modificar sus esquemas cognitivos a partir de unos conocimientos previos, obteniendo así un aprendizaje significativo
(Romero y Quesada, 2014). Dentro de la amplia gama de TIC, encontramos herramientas de comunicación síncronas y asíncronas como el chat y el foro, herramientas de gestión como la plataforma virtual, marcadores sociales, y herramientas de creación como blogs o wikis (Guerra-Martín, 2015). Debido a la gran variedad y disponibilidad de herramientas gestoras de contenidos, el alumnado en su actividad procedimental no necesita adquirir la totalidad de conocimientos del currículo, sino que debe ser capaz de conocer y usar fuentes de información, y adaptarse a nuevos entornos de aprendizaje (Siemens, 2004). Para Siemens, autor de la teoría del conectivismo, el aprendizaje es un proceso continuo que dura toda la vida en el que el procesamiento de la información se realiza fuera de la mente humana, en la tecnología. Así, la persona aprende a través de comunidades de práctica, redes personales y realización de tareas relacionadas con trabajos.

El aprendizaje en la Formación Profesional se centra en la habilidad de aplicar los conceptos a la práctica real (Isus y Roure, 2012; Guerra-Martín, 2014). Teniendo en cuenta la teoría del conectivismo de Siemens (2004), el alumnado desarrolla el conocimiento y la comprensión a través de la experiencia y la reflexión, conectando con su red de aprendizaje y tomando decisiones. Atendiendo estos principios, la Formación Profesional no trata de transmitir contenidos cerrados o aquello que el profesorado estima oportuno, sino que los contenidos que se ofrecen son solo la base del aprendizaje. Se parte de la idea de que el alumnado debe ser capaz de buscar, ampliar, desarrollar, sintetizar, interactuar y reflexionar para aprender. En este sentido, una forma de aprendizaje que fomenta estas capacidades es el aprendizaje inverso o flipped classroom. Se trata de un enfoque pedagógico que transfiere fuera del aula el trabajo de determinados procesos de aprendizaje y utiliza el tiempo de clase, apoyándose en la experiencia del docente, para facilitar y potenciar otros procesos de adquisición y práctica de conocimiento (Bergmann y Sams, 2012). El término flipped classroom fue introducido por Bergmann y Sams (2012), conscientes de que algunos alumnos no asistían a clase por diversas razones, por lo que impulsaron la grabación y distribución de vídeos. Usando este modelo ambos profesores se dieron cuenta del potencial pedagógico para aprender, de una forma más individualizada, las necesidades de aprendizaje de cada alumno (Tourón y Santiago, 2015). Bajo este modelo el profesorado puede distribuir contenido didáctico al alumnado a través de recursos digitales $\mathrm{y}$, por su parte, el alumnado puede acceder a él donde y cuando quiera 
mientras tenga un dispositivo electrónico y conexión a Internet.

Estos modelos de aprendizaje integran las TIC como recurso pedagógico y no meramente técnico. Este uso pedagógico otorga a la enseñanza innovación y mejora potencial. Entre otros, los principales beneficios, son: 1. Permitir a los docentes dedicar más tiempo a la atención a las diferencias individuales. 2. Ser una oportunidad para que el profesorado pueda compartir información y conocimientos entre sí, con el alumnado, las familias y la comunidad. 3. Proporcionar al alumnado la posibilidad de volver a acceder, tantas veces como sea necesario, a los mejores contenidos generados o facilitados por sus profesores. 4. Crear un ambiente de aprendizaje colaborativo en el aula. 5. Involucrar a las familias desde el inicio del proceso de aprendizaje (Tourón y Santiago, 2015).

En base a lo anteriormente referido, es por lo que nos propusimos como objetivos poner en marcha el aprendizaje inverso en el aula durante el curso $2014 / 2015$ y conocer las opiniones del proceso de aprendizaje de los alumnos de la asignatura Dotación Sanitaria dentro del Ciclo Formativo de Grado Medio (CFGM) de Técnico en Emergencias Sanitarias de Sevilla (España).

\section{MATERIAL Y MÉTODO}

En la asignatura Dotación Sanitaria es obligatorio el uso de las TIC, ya que los alumnos están matriculados en un CFGM de modalidad semipresencial, donde se utiliza la plataforma Moodle, en la que se inserta el material didáctico para ser trabajado por el alumnado antes de las clases presenciales. Además, se cuenta también con diversos recursos en Internet (blogs, wikis, bases de datos, repositorios de vídeos, imágenes, presentaciones...).

Entre los recursos tecnológicos disponibles en Internet, se seleccionan los siguientes: 1. Herramienta de gestión: plataforma Moodle (anexo 1). 2. Herramienta de comunicación asíncrona: foro. 3. Herramienta de administración: Symbaloo (anexo 2). 4. Herramienta de edición de vídeo y screencast: Knovio (anexo 3). El proceso de aprendizaje se desarrolló usando las herramientas tecnológicas citadas anteriormente.

Esta experiencia educativa mediante la metodología del aprendizaje inverso se llevó a cabo durante el curso 2014/2015. Todos los alumnos tenían dispositivos electrónicos y conexión a Internet en sus domicilios, por lo que el uso de las
TIC y la aplicación del aprendizaje inverso fueron posibles durante el curso.

El profesorado, tras programar las actividades del módulo profesional, diseñó los materiales didácticos a través del screencast o grabación de vídeo con narración. Usó la herramienta Knovio con la que, a través de presentaciones en formato PowerPoint, explicaba cada contenido con grabación de audio y vídeo de forma simultánea. En cada diapositiva se insertaron textos, imágenes y vídeos acompañados de la narración continua del profesorado. Este screencast fue colgado en la plataforma Moodle junto con los contenidos del temario en PDF. De esta forma, el alumnado tenía la opción de buscar información en los contenidos, además de usar la herramienta de administración Symbaloo, que es un marcador social con el que se consigue una organización de sitios web. Si, aun así, el alumnado seguía teniendo dudas, tenía disponible en la plataforma la herramienta de comunicación asíncrona foro, con la que podía plantear dudas antes de la clase presencial. Este proceso ocurre cuatro días antes de la explicación y corrección de actividades en el aula. Así, se permite que el alumnado trabaje los contenidos antes de llegar a la clase, siendo el aprendizaje autónomo y colaborativo. Esta personalización del aprendizaje ofrece motivación al alumnado para aprender, ahorro de tiempo en clase, concreción en la resolución de dudas y protagonismo del alumnado en su proceso de aprendizaje (Tourón y Santiago, 2015).

Una vez finalizada la asignatura, se llevó a cabo un estudio descriptivo y transversal (Polit y Hungler, 2000; Hernández et al., 2006; Cea, 2009), para conocer las opiniones de los alumnos sobre el proceso de aprendizaje. La muestra estuvo conformada por los trece estudiantes (100\%) que estaban matriculados en la asignatura. Se realizó un cuestionario de elaboración propia. El instrumento de medición quedó integrado por doce ítems con seis opciones de respuesta tipo Likert: 1. No lo sé. 2. Totalmente en desacuerdo. 3. En desacuerdo. 4. Indiferente. 5. De acuerdo. 6. Totalmente de acuerdo.

Los ítems fueron los siguientes: 1. El uso de las Tecnologías de la Información y Comunicación (TIC) mejora en general mi rendimiento formativo. 2. Comprendo mejor los contenidos cuando hay apoyo de un vídeo y/o imagen. 3. En casa, con apoyo de las TIC, estudio y asimilo mejor los contenidos. 4. Tras ver los vídeos enviados por el profesor, buscar información en mi casa y preguntar las dudas después en el aula, aprendo. 5. La implementación del modelo de aprendizaje inverso me da mayor autonomía en el proceso de 
aprendizaje. 6. El aprendizaje inverso me parece motivador. 7. El aprendizaje inverso es más motivador que una clase expositiva en el aula. 8. El profesorado está más atento al alumnado cuando se aplica el modelo de aprendizaje inverso. 9. Con la aplicación del aprendizaje inverso, el aprendizaje es más individualizado. 10. Aprendo mejor haciendo las actividades con apoyo de las TIC que en las clases magistrales. 11. El aprendizaje inverso se adapta a mi ritmo y estilo de aprendizaje. 12. El aprendizaje inverso me permite tener más tiempo para resolver las dudas en clase.

La recogida de datos se llevó a cabo en el aula, de manera autoadministrada, durante el mes de marzo de 2015. El tiempo medio de cumplimentación del cuestionario fue de treinta minutos. Con el objetivo de respetar la confidencialidad de los datos y los principios bioéticos, se informó a los participantes del objetivo del estudio y se les solicitó el consentimiento informado. El estudio fue previamente aprobado por la jefatura de estudios y servicio de orientación pedagógica del centro educativo.
En la muestra de estudio ( $\mathrm{N}=13)$ un $70 \%$ (9) ha accedido a Formación Profesional por primera vez, un $62 \%$ (8) no tiene formación ni experiencia relacionada con el sector de la sanidad, un $77 \%$ (10) se ha matriculado al CFGM para encontrar un empleo mejor al que tienen en la actualidad, un $62 \%$ (8) están desempleados. Respecto al sexo, un $84 \%$ (11) eran hombres.

La edad media es de 37,7 años y la desviación típica de 8,18. Por grupos de edades: un $22 \%$ (3) de los alumnos tienen entre 20 - 29 años (22\%), un $39 \%$ (5) entre 30 - 39 años y otro $39 \%$ (5) tienen más de 40 años.

En la tabla 1 se presentan los resultados del cuestionario de opinión de los alumnos. En el ítem 12 (El aprendizaje inverso me permite tener más tiempo para resolver las dudas en clase) el $100 \%$ de los alumnos estuvo de acuerdo y totalmente de acuerdo. Además, ningún estudiante ha valorado negativamente (totalmente en desacuerdo y en desacuerdo) a ningún ítem, ni ha dejado ninguna pregunta sin contestar del cuestionario.

\section{RESULTADOS}

Tabla 1. Resultados del cuestionario de opinión de los alumnos de la asignatura Dotación Sanitaria. Curso 2014/2015. 1. No lo sé. 2. Totalmente en desacuerdo. 3. En desacuerdo. 4. Indiferente. 5. De acuerdo. 6. Totalmente de acuerdo.

\begin{tabular}{c}
\hline Ítems \\
\hline 1. El uso de las TIC mejora mi rendimiento formativo \\
2. Comprendo mejor los contenidos con apoyo de vídeo \\
y/o imagen
\end{tabular}

3. En casa, con apoyo de las TIC, estudio y asimilo mejor los contenidos

4. Aprendo tras ver los vídeos enviados por el profesor, buscar información en mi casa y preguntar las dudas después en el aula

5. La implementación del modelo de aprendizaje inverso me da mayor autonomía en el proceso de aprendizaje

6. El aprendizaje inverso me parece motivador

7. El aprendizaje inverso es más motivador que una clase expositiva en el aula

8. El profesorado está más atento al alumnado cuando se aplica el modelo de aprendizaje inverso

\begin{tabular}{cccccc}
$\mathbf{1 .}$ & $\mathbf{2 .}$ & $\mathbf{3}$ & $\mathbf{4 .}$ & $\mathbf{5 .}$ & $\mathbf{6 .}$ \\
$\mathbf{N}$ & $\mathrm{N}$ & $\mathrm{N}$ & $\mathrm{N}$ & $\mathbf{N}$ & $\mathbf{N}$ \\
$(\%)$ & $(\%)$ & $(\%)$ & $(\%)$ & $(\%)$ & $(\%)$ \\
\hline 0 & 0 & 0 & 0 & 5 & 8 \\
$(0)$ & $(0)$ & $(0)$ & $(0)$ & $(38,5)$ & $(61,5)$ \\
0 & 0 & 0 & 0 & 0 & 13 \\
$(0)$ & $(0)$ & $(0)$ & $(0)$ & $(0)$ & $(100)$ \\
0 & 0 & 0 & 2 & 4 & 7 \\
$(0)$ & $(0)$ & $(0)$ & $(15,4)$ & $(30,8)$ & $(53,8)$ \\
& & & & & \\
0 & 0 & 0 & 2 & 4 & 7 \\
$(0)$ & $(0)$ & $(0)$ & $(15,4)$ & $(30,8)$ & $(53,8)$ \\
& & & & & \\
0 & 0 & 0 & 1 & 10 & 2 \\
$(0)$ & $(0)$ & $(0)$ & $(7,7)$ & $(76,9)$ & $(15,4)$ \\
& & & & & \\
0 & 0 & 0 & 0 & 4 & 9 \\
$(0)$ & $(0)$ & $(0)$ & $(0)$ & $(30,8)$ & $(69,2)$ \\
0 & 0 & 0 & 3 & 7 & 3 \\
$(0)$ & $(0)$ & $(0)$ & $(23,1)$ & $(53,8)$ & $(23,1)$ \\
0 & 0 & 0 & 0 & 5 & 8 \\
$(0)$ & $(0)$ & $(0)$ & $(0)$ & $(38,5)$ & $(61,5)$ \\
& & & & & \\
& & & & &
\end{tabular}


9. Con la aplicación del aprendizaje inverso, el aprendizaje es más individualizado

10. Aprendo mejor haciendo las actividades con apoyo de las TIC que en las clases magistrales

11. El aprendizaje inverso se adapta a mi ritmo y estilo de aprendizaje

12. El aprendizaje inverso me permite tener más tiempo para resolver las dudas en clase

\section{DISCUSION}

En cuanto al ítem 1: El uso de la Tecnología de la Información y Comunicación (TIC) mejora en general mi rendimiento formativo, se observa que la totalidad del alumnado afirma estar de acuerdo y totalmente de acuerdo. Este resultado está en concordancia con otros estudios donde han analizado también, las opiniones de los alumnos (Santoveña, 2007; Henríquez, 2008; Guerra-Martín et al, 2013; Guerra-Martín et al, 2014; GuerraMartín, 2015).

Respecto al ítem 2 sobre la comprensión de los contenidos con el apoyo de una imagen o vídeo, todo el alumnado está de acuerdo y totalmente de acuerdo con que mejora la comprensión con el uso de recursos multimedia. El alumnado dispone de diferentes fuentes de información, y aprende de manera informal a través de las opiniones de sus compañeros, profesorado y personas que publican en sitios web (Marcelo, 2011). Además de la ayuda pedagógica, puede ser debido a la posibilidad que tiene el alumnado de volver a acceder al recurso facilitado por su profesorado tantas veces como sea necesario (Tourón y Santiago, 2015), ya que un vídeo muestra un proceso real y acerca al alumnado a su futura actividad profesional.

En el ítem 3: En casa, con apoyo de las TIC, estudio y asimilo mejor los contenidos, casi el total de alumnos han afirmado estar de acuerdo y totalmente de acuerdo. En este sentido, decir que las nuevas generaciones crecen usando TIC, en contra de las generaciones más adultas que generalmente tienen poco o ningún manejo. Los recursos tecnológicos pueden ser usados como estrategia para recopilar, interpretar y organizar la nueva información (Nicasio, 2001), pero existen otras estrategias que se adaptan al estilo de aprendizaje de cada alumno.

En cuanto al ítem 4: Tras ver los vídeos enviados por el profesor, buscar información en mi casa y preguntar las dudas después en el aula, aprendo, casi la totalidad de los estudiantes han afirmado estar de

$\begin{array}{cccccc}0 & 0 & 0 & 1 & 3 & 9 \\ (0) & (0) & (0) & (7,7) & (23,1) & (69,2) \\ 0 & 0 & 0 & 0 & 3 & 10 \\ (0) & (0) & (0) & (0) & (23,1) & (76,9) \\ 0 & 0 & 0 & 5 & 5 & 3 \\ (0) & (0) & (0) & (38,5) & (38,5) & (23,1) \\ 0 & 0 & 0 & 0 & 2 & 11 \\ (0) & (0) & (0) & (0) & (15,4) & (84,6)\end{array}$

acuerdo y totalmente de acuerdo. Tenemos que decir que una estrategia democrática y colaborativa aumenta y mantiene la motivación, como sucede con el uso pedagógico de las TIC.

Siguiendo el aprendizaje inverso como principal metodología, el profesorado asume un rol de facilitador en la realización de las tareas, buscando la participación del alumnado en la toma de decisiones (Cuadrado, 2009; Guerra-Martín et al., 2013; Guerra-Martín, 2014).

El estudio demuestra que todos los alumnos afirman estar de acuerdo y totalmente de acuerdo, con el ítem 5: La implementación del modelo de aprendizaje inverso da mayor autonomía en el proceso de aprendizaje. De este modo, se corrobora el análisis realizado por los autores Bergmann y Sams (2012), que tratan el aprendizaje inverso como una transferencia fuera del aula del trabajo de determinados procesos de aprendizaje, utilizando el tiempo de clase y apoyándose en la experiencia del docente, para facilitar y potenciar otros procesos de adquisición y práctica de conocimiento. La autonomía y el protagonismo en el proceso de aprendizaje recaen en el alumnado, siendo el profesorado su guía (Guerra-Martín et al., 2014; Guerra-Martín, 2015).

La totalidad de los alumnos afirma estar de acuerdo y totalmente de acuerdo con los ítems 6 y 7 , sobre que el aprendizaje inverso les parece motivador, y que es más motivador que una clase expositiva en el aula. El alumnado está acostumbrado por su experiencia formativa a recibir una lección magistral apoyada de un libro de texto, con el que luego realiza la tarea propuesta por el profesorado. Este modelo rompe la metodología tradicional recibida durante años y aunque resulta difícil instaurarse en el aula, es más motivadora y beneficiosa a largo plazo para los estudiantes (Lozano, 2000; Núñez, 2009; Bergmann y Sams, 2012; Tourón y Santiago, 2015).

En cuanto a los ítems 8 y 9: El profesorado está más atento al alumnado cuando se aplica el modelo de aprendizaje inverso y con la aplicación del aprendizaje inverso, y El aprendizaje es más individualizado. Todos los alumnos del estudio usan 
las TIC como medio de gestión del conocimiento, y es en el aula donde aprenden rectificando los errores, discutiendo con los compañeros, preguntando al profesor y tomando decisiones (Guerra-Martín et al., 2013; Guerra-Martín, 2014).

Con respecto al ítem 10: Aprendo mejor haciendo las actividades con apoyo de las TIC que en las clases magistrales, todos los alumnos están de acuerdo y totalmente de acuerdo. Esta buena valoración puede deberse a que, al ser una formación mayormente práctica, en los recursos audiovisuales el alumnado identifica los protocolos y procedimientos a seguir. Es un recurso que acerca la realidad al aula (Cuadrado, 2009).

En cuanto al ítem 11 el alumnado afirma que el aprendizaje inverso se adapta a su ritmo y estilo de aprendizaje, en este sentido, Amador (2011) plantea que no hay una única ruta de aprendizaje, sino que las posibilidades pueden ser múltiples, dependiendo del contexto, del tiempo y, sobre todo, de las características, intereses y necesidades de las personas implicadas en la formación. El conjunto de estos factores da lugar a un estilo de aprendizaje personal.

Para finalizar, con el ítem 12 sobre el aprendizaje inverso permite tener más tiempo para resolver las dudas en clase, la totalidad del alumnado está de acuerdo y totalmente de acuerdo. Este resultado verifica lo que plantea la bibliografía consultada, destacando que el aprendizaje inverso es un modelo eficiente. Con un menor gasto de recursos materiales y tiempo, se puede aprender mejor y ofrecer una enseñanza individualizada y de calidad (Bergmann y Sams, 2012; Tourón y Santiago, 2015).

En cuanto a las limitaciones de este estudio, decir que la muestra estudiada ha sido pequeña, que el alumnado tiene un perfil heterogéneo y que el tiempo de estudio ha sido limitado.

\section{CONCLUSIONES}

Los recursos pedagógicos que ofrece Internet son numerosos. El alumnado reconoce que el aprendizaje inverso es más motivador que el tradicional, ya que promueve la investigación y reflexión, y mejora el rendimiento formativo. El modelo tradicional no permite atender a las singularidades de los alumnos y practicar un aprendizaje individualizado, ya que no todos los estudiantes tienen las mismas necesidades, ni el mismo nivel y velocidad de aprendizaje. Es por lo que, este enfoque tradicional ofrece pocas oportunidades para la diferenciación, creatividad, desarrollo de inteligencias múltiples y fomento de la motivación en el alumnado.

Por todo ello, se propone la puesta en marcha e implementación del aprendizaje inverso o flipped classrrom como modelo pedagógico. Para llevar a cabo este aprendizaje inverso se necesita una tecnología apropiada y un diseño pedagógico centrado en promover el trabajo autónomo del alumno. El aprendizaje inverso pone el peso de la acción de aprender en los estudiantes, siendo estos los protagonistas de su propio aprendizaje. Además, pone de relieve la función de los tutores, como guías y facilitadores del proceso de aprendizaje, dentro de un entorno enriquecido tecnológicamente y teniendo en cuenta las características y necesidades de los estudiantes.

\section{REFERENCIAS}

Amador, L., Monreal, M.C., y Marco, M.J. (2001). El adulto: etapas y consideraciones para el aprendizaje. Eúphoros, 3, 97-112. Recuperado de: http://dialnet.unirioja.es/servlet/articulo?codigo=118306 3

Ausubel P. (1986). Psicología educativa: un punto de vista cognoscitivo. México: Editorial Trillas.

Bergmann, J., y Sams, A. (2012). Flip your Classroom: Reach Every Student in Every Class Every day. Washington, DC: ISTE; and Alexandria, VA: ASCD.

Cea, M. A. (2009). Metodología cuantitativa. Estrategias y técnicas de investigación social. $3^{a}$ reimpresión. Madrid: Síntesis Sociológica.

Cuadrado, B. (2009). El profesorado como líder grupal. Revista Digital Innovación y Experiencias Educativas 19, 1-9. Recuperado de: http://www.csicsif.es/andalucia/modules/mod_ense/revista/pdf/Numero _19/BEGONA_CUADRADO_1.pdf

De la Torre, F. (2000). Relaciones humanas en el ámbito laboral. México: Editorial Trillas.

García, F. J. y Doménech, F. (2002). Motivación, aprendizaje y rendimiento escolar. Revista Electrónica de Motivación y Emoción, 1(6), 24-36. Recuperado de: http://www.revistadocencia.cl/pdf/20100728164200.pdf

Gómez, M. y Vidangos, K. (2006). El impacto de las tecnologías de información y comunicación en el proceso enseñanza-aprendizaje. Universidad del Valle.

Guerra-Martín M. D., Lima-Serrano, M., ZambranoDomínguez, E. M. y Fernández-Rodríguez, V. (2013). Evaluación de una intervención sobre búsquedas de información científica para estudiantes de enfermería. Texto \& Contexto- Enfermagem 22 (3): 619-628. doi: http://dx.doi.org/10.1590/S0104-07072013000300007

Guerra-Martín MD. (2014). Tutoring as a way of achieving employability for nursing students at the University of Seville. Procedia-Social and Behavioral Sciences, 139: 479-486. Higher Education: The challenge of employability (XIII International Congress of Educational Theory). doi: 10.1016/j.sbspro.2014.08.049

Guerra-Martín, M. D, Lima-Serrano, M., ZambranoDomínguez, E. M. y Lima-Rodríguez, J. S. (2014). ¿Es efectivo el aprendizaje sobre alfabetización en información para estudiantes de enfermería? Enfermería 
Global $13 \quad$ (4): $\quad 90-102 . \quad$ doi: http://dx.doi.org/10.6018/eglobal.13.4.182541

Guerra-Martín, M. D. (2015). Características de las tutorías realizadas por el profesorado de los estudios de Enfermería de la Universidad de Sevilla. Sevilla: Punto Rojo Libros.

Gureckis, T. M. y Markant, D. B. (2012). Self-Directed Learning: a Cognitive and Computational Perspective. Perspectives on Psychological Science, 7(5), 464-481. doi:10.1177/1745691612454304.

Henríquez, G. (2008). La tutoría electrónica aplicada en los proyectos de Investigación en salud. Revista Iberoamericana de Educación a Distancia, 12 (1): 7983.

Hernández, R., Fernández-Collado, C. y Baptista, P. (2006). Metodología de la investigación, 4 ed. México: McGraw-Hill.

Isus, S. y Roure, J. (2012). Dispositivos de institucionalización y dinámicas de profesionalización de la formación en alternancia en Educación Superior. En S. Carrasco (Direct.), La universidad: una institución de la sociedad (pp.49-52). Barcelona: Ediciones Octaedro.

Julián, J. F. y Narváez, C.G. (2010). Motivación y Ejercicio Docente en la Educación Superior: Una Aproximación Conceptual. Educere, 48, 21-27. Recuperado de: http://www.redalyc.org/articulo.oa?id=35616720003

Lozano, L. M., Fernández, L., García-cueto, E. y Gallo, P. (2000). Relación entre motivación y aprendizaje, Psicothema, 12(2), 344-347. Recuperado de: http://www.psicothema.com/pdf/579.pdf

Marcelo, C. (2010). Autoformación para el siglo XXI. En J. Gairín (Coord.). Nuevas estrategias formativas por las organizaciones (pp.141-170). Madrid: Wolters Kluwer.

Marcelo, C. (2011). Aprendizaje informal. No es oro todo lo que reluce. [Mensaje del Blog CUED. Cátedra UNESCO de Educación a Distancia de la UNED] Recuperado 26 de julio de 2015 a partir de: http://blogcued.blogspot.com.es/2011/09/aprendizajeinformal-no-es-oro-todo-lo.html

Moreira, M. C. (2012). ¿Al final qué es aprendizaje significativo? Qurriculum: Revista de teoría, investigación y práctica educativa, 25, 29-56. Recuperado

http://publica.webs.ull.es/upload/REV\%20QURRICUL $\mathrm{UM} / 25 \% 20-\% 202012 / 02 . p d f$

Nicasio, J. (2001). Estilo de aprendizaje y su evaluación. Educación, desarrollo y diversidad, 3, 27-38. Recuperado de: http://dialnet.unirioja.es/servlet/articulo?codigo=197463 7

Núñez, J. C. (2009). Motivación, aprendizaje y rendimiento académico. En Actas Do X Congresso Internacional Galego-Português de Psicopedagogia. (41-67). Braga: Universidad do Minho. Recuperado de http://www.educacion.udc.es/grupos/gipdae/documentos /congreso/Xcongreso/pdfs/cc/cc3.pdf

Polit D.F. y Hungler B.P. (2000). Investigación Científica en Ciencias de la Salud. $6^{a}$ ed. McGraw-Hill Interamericana. México.

RAE (2001). Diccionario de la Lengua Española. (22 ${ }^{\mathrm{a}}$ ed.) Real Academia Española. Madrid.

Romero, M. y Quesada, A. (2014). Nuevas Tecnologías y aprendizaje significativo de las ciencias. Enseñanza de las Ciencias, 32(1), 101-115. Recuperado de:
http://www.raco.cat/index.php/Ensenanza/article/view/2 $87510 / 375663$

Santoveña, S.M. (2007). La tutorización de los cursos virtuales de la diplomatura de educación social de la UNED. Revista Electrónica Teoría de la Educación. Educación y Cultura en la Sociedad de la Información, vol. Extraordinario, 212-224. Recuperado de: http://www.usal.es/teoriaeducacion

Siemens, G. (2004). Conectivismo: una teoría de aprendizaje para la era digital. Recuperado de: http://d.scribd.com/docs/1yhhhthpoaervbohwzkc.pdf

Tourón, J. y Santiago, R. (2013). Atención a la diversidad y desarrollo del talento en el aula. El modelo DT-PI y las tecnologías en la implantación de la flexibilidad curricular y el aprendizaje al propio ritmo. Revista Española de Pedagogía, 256, 441-459.

Tourón, J. y Santiago, R. (2015). El modelo Flipped Learning y el desarrollo del talento en la escuela. Revista de Educación, 368, 196-231. doi:10.4438/1988-592X-RE2015-368-288.

\section{ANEXOS}

Anexo 1. Plataforma virtual tipo Moodle del Centro Oficial de Formación Grado Superior Cesur.

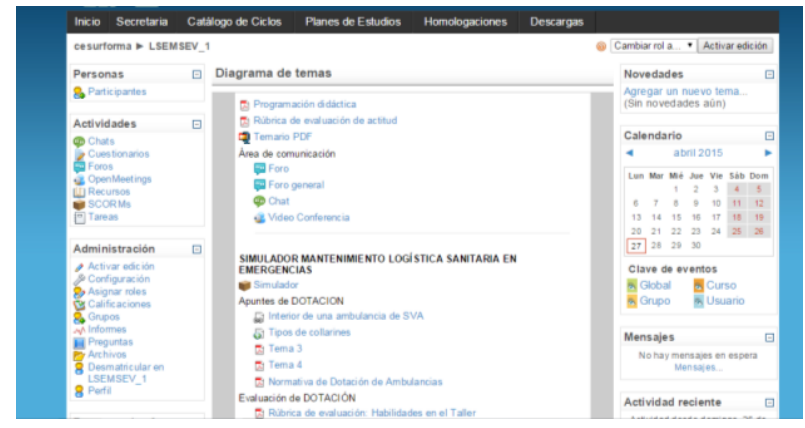

Fuente: http://www.cesurformaciononline.com/moodle/

Anexo 2. Herramienta de administración Symbaloo.

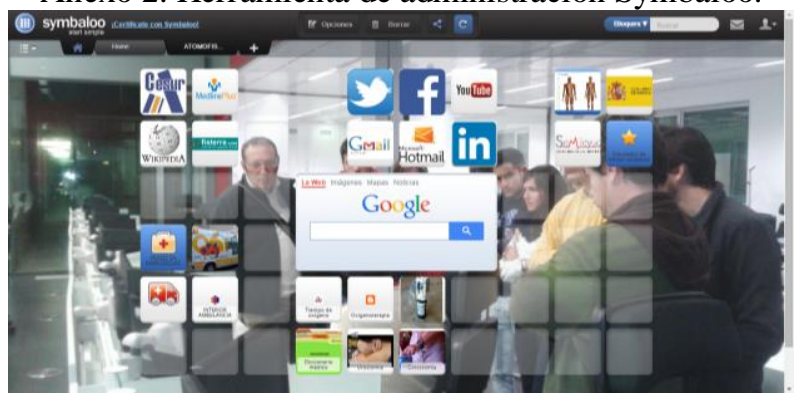

Fuente:

http://www.symbaloo.com/home/mix/13eOcLjTaQ 
Anexo 3. Herramienta de edición de vídeo con narración Knovio

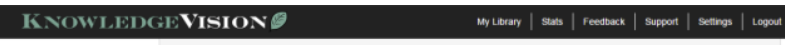

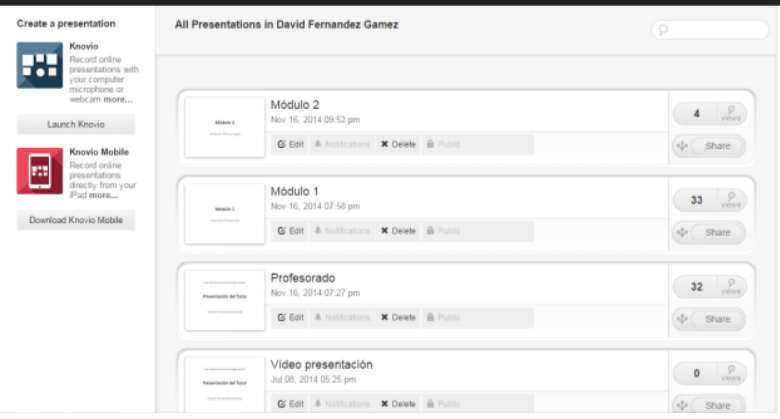

Fuente: http://www.knovio.com/ 